\title{
Survey of Glyph-based Visualization Techniques for Spatial Multivariate Medical Data
}

\author{
Timo Ropinski, Steffen Oeltze and Bernhard Preim
}

\begin{abstract}
In this survey article, we review glyph-based visualization techniques which have been exploited when visualizing spatial multivariate medical data. To classify these techniques, we derive a taxonomy of glyph properties that is based on classification concepts established in information visualization. By considering both the glyph visualization as well as the interaction techniques that are employed to generate or explore the glyph visualization, we are able to classify glyph techniques into two main groups: those supporting pre-attentive and those supporting attentive processing. With respect to this classification, we review glyph-based techniques described in the medical visualization literature. Based on the outcome of the literature review, we propose design guidelines for glyph visualizations in the medical domain.
\end{abstract}

Keywords: Glyphs, medical visualization, multivariate data

\section{Introduction}

Data sets acquired in the medical domain, contain a multitude of information that provides a huge potential for diagnosis and individualized therapy planning. However, to exploit this potential, the data needs to be interpreted efficiently. In the past, mainly the increasing resolution of the scalar volume data sets posed a challenge for medical visualization. Algorithms had to be developed in order to extract and emphasize structures of interest. Today, also the multiple variables which can be derived from different modalities or time steps, pose a challenge when interpreting visualizations. When dealing with 3D data sets, as done in this article, additional problems arise, as for instance occlusion handling or choosing an appropriate projection.

One essential example, where multivariate data sets accrue is the 4D ultrasound acquisition of the human heart, which recently became a routine proceeding. With this method it is possible to derive multiple variables during one examination, i. e., information regarding the structure of the heart as well as direction and amount of blood flow. Another example are data sets acquired with multimodal medical scanners. When, for instance combining positron emission tomography (PET) with computed tomography (CT) it becomes possible to obtain an integrated visualization of metabolism activity within a high resolution structural context. To address the visualization challenges posed by these multivariate medical data sets, glyph-based techniques are a viable option $[1,2]$.

Glyphs are considered as symbolic or iconic representations of one or more variables of a data set. They are usually geometric objects, whose visual representation can be altered through changing the glyph properties. By using a parameter mapping function (PMF), the variables that have to be represented can be associated with one or more properties of a glyph, e. g., shape, size or color. The parameter mapping can either be continuous or discrete. When interpreting glyph-based visualizations, the visual representation of individual glyphs as well as the overall structure given by the arrangement of all glyphs can be exploited $[1,2]$. Bürger and Hauser describe glyphs as just one visualization technique for multi-variate data [2]. They mention the benefit that a large number of data dimensions can be incorporated and the mapping can be used to reflect semantics. Another advantage is the ability to combine glyphs with other visualization techniques. On the other hand continuity is not given, and thus the data set depicted by the glyphs is only visualized in a discrete manner. Therefore, the visualization designer should carefully review the visualization requirements before using glyphs.

In this article, we describe and classify the glyph-based techniques exploited when visualizing spatial multivariate medical data. Ward classifies different glyph placing strategies and proposes rules for their usage in the context of information visualization [1]. In this article, we restrict ourselves to the review of glyph-based visualization techniques for medical data sets. In contrast to most applications in information visualization, in medical visualization the spatial embedding of the glyphs is of major interest. In almost every case, the data is regularly sampled, whereby for each sampling point either one or a set of scalar variables are present. We propose a taxonomy of glyph properties specifically designed for medical visualization that is based on findings from the perception literature. Rather than classifying glyph techniques with respect to their technical properties, we address the way they communicate the information to be visualized. This is essential since in medical diagnosis time is usually the limiting factor, and thus an efficient communication is essential. The described taxonomy extends the taxonomy proposed by Ropinski and Preim [3]. In particular, we now also consider the findings from higher-order tensor glyphs which play an important role in the literature. Further- 
more, we introduce a more strict differentiation between preattentive and attentive glyph properties. Thus, we introduce the appearance property as a pre-attentive glyph property. These new considerations lead to a concise set of usage guidelines for glyph-based medical visualizations, which we believe has the potential to improve future glyph applications in the medical domain.

After discussing our glyph taxonomy in the next section, we will describe the usage of glyphs in selected medical application areas in Section 3. There, we will cover visualization of cardiac MRI data, and diffusion weighted MRI (DW-MRI) data in general. Based on the best practice we have identified in our literature review, we propose some usage guidelines in Section 4 , before concluding the paper in Section 5.

\section{Glyph-Based Medical Visualization Taxonomy}

Efficiency as well as accuracy of information processing play a crucial role in clinical practice, and therefore an intuitive association between the visualization of data and their meaning is important. Glyph representations are not only used in medical research. It has also been shown in user studies that their use can be beneficial in the medical diagnosis [4]. However, due to the time constraints during a diagnosis, it is of great interest that the most important of these associations can be done in a very short time frame. Semiotic theory concentrates on signs and how they convey meaning. According to semiotic theory, stimuli are processed in two phases [5]. The first phase is the pre-attentive one, where impulses are perceived in parallel and instantly (within $250 \mathrm{~ms}$ ) as one entity. Within this phase facts that can be easily perceived are extracted, for example the overall structure of a visualization, strong differences in shape, and strong differences in color [6]. In the second more goal-driven phase of the perception process the visualization is analyzed sequentially, i. e., parts of the visualization are identified and observed more detailed, one after another. Due to the conscious nature of this phase, we refer to it as attentive phase in the remainder of this article. According to Treisman, the initial pre-attentive phase is the major step towards improved comprehension [7]. This theory is supported by recent findings in fMRI-based brain research [8]. Thus, visualization designers should choose the pre-attentive stimuli wisely in order to communicate the desired information effectively. In fact, most glyph-based visualizations exploit pre-attentive stimuli, such that the distribution of glyph size, shape and color as well as the glyph aggregation aid visual comprehension. Attentive stimuli are mainly considered in glyph-based visualizations by exploiting interaction metaphors. Thus, after the pre-attentively perceivable information is extracted, the user can interactively explore the glyph visualization. This explorative process is also reflected in Shneidermans overview, zoom, filter out, details-ondemand concept that describes a general explorative task to be performed through these four steps [9].

Therefore, our taxonomy that is based on the preliminary work presented in [3], allows to distinguish between preattentive stimuli (Section 2.1) and attentive stimuli processing through appropriate interaction metaphors (Section 2.2). To be

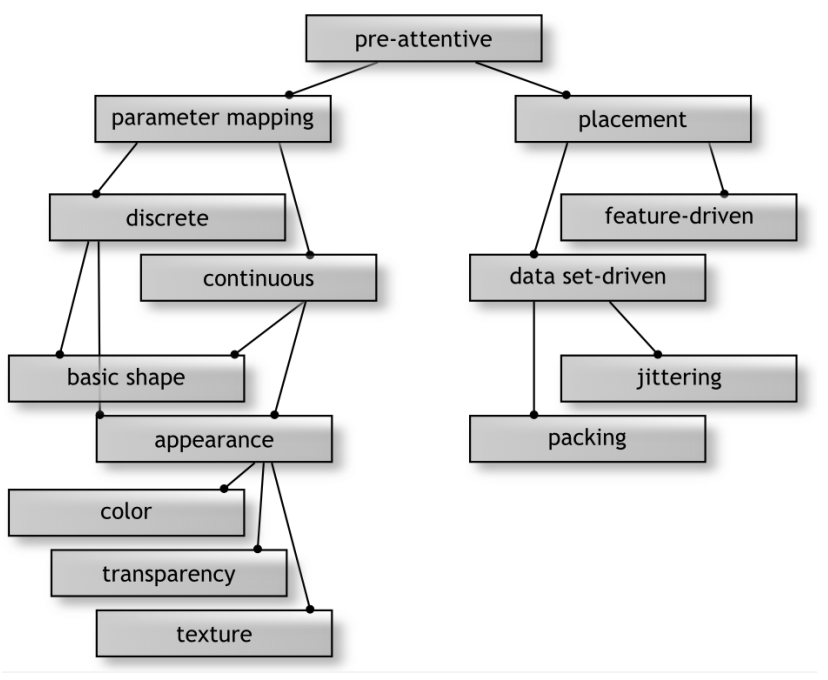

Figure 1: We consider parameter mapping and glyph placement as the two main groups of glyph properties, which are perceived pre-attentively.

able to incorporate also future glyph-based techniques, we do not consider entire glyph visualizations, but regard different aspects as glyph shapes, parameter mapping or placement, which are integrated into the taxonomy.

\subsection{Pre-Attentive Stimuli}

Pre-attentive stimuli, relevant for glyph-based visualization, can be classified with respect to glyph shapes as well as glyph appearance, such as color, transparency and texture (see Fig. 1). Furthermore, the glyph placement strategies have an influence, since the spatial distribution can also be perceived preattentively. These pre-attentive stimuli can be exploited only in order to extract basic features of objects in the display which include colors, closure, line ends, contrast, tilt, curvature, and size [7]. A quantitative analysis occurs in the attentive processing phase which we address in Section 2.2.

\subsubsection{Glyph Shape}

The shape of a glyph is the main characteristic and it is important that it can be perceived easily and unambiguously [10]. Since the perception of shapes as well as spatial relationships is more accurate than the perception of quantities depicted by colors [11], the glyph shapes, and also glyph placements (see Section 2.1.3), are primarily used to convey information. We distinguish two main groups of glyph shapes:

1. Basic glyph shapes are geometric objects which can be modified by changing their geometric properties, such as size or orientation. Wide-spread examples are spheres, cuboids, and ellipsoids.

2. Composite glyph shapes are composed of the basic glyph shapes. Composite glyph shapes require a more specialized mapping function, i. e., parameters usually cannot be mapped to geometric properties as radius or length. They are often used to display multivariate data, whereby each variable can be communicated using another property of the composed building blocks. 


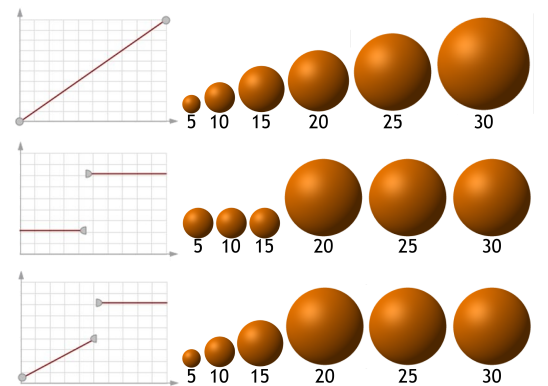

Figure 2: The mapping function (left) maps a parameter to the size of a glyph (right). Depending on the continuity of the mapping function, its reconstruction can be rather challenging without having a legend.

Regarding the complexity of composite glyph shapes, we assume that mainly basic glyph shapes benefit from the improved perception of shapes and spatial relationships in the pre-attentive phase, and consequently discuss composite glyph shapes within Section 2.2.

Continuous and discrete mapping. For both basic and composite glyph shapes either a continuous or a discrete mapping can be used in order to communicate information based on the glyph's properties. Ropinski et al. described variations of mapping functions [12]. They point out that also a step mapping function can be used in order to allow a better differentiation of the values to be visualized. Such a mapping is shown in Fig. 2, where glyphs representing different data values are shown next to the respective PMF. The mapping type should always be application-driven. For instance, to support the decision process in therapy and diagnosis, a discrete mapping might be appropriate, where color could be used to classify tissue states, e.g., benign suspicious, malign. In contrast, when quantifying perfusion, the whole range of scalar values might be of interest. When using a spherical glyph, such a scalar data value can be continuously mapped to its radius (recall Fig. 2). Discrete mappings often better support a quantitative analysis. Since a perspective projection is used in many cases, the size of a glyph cannot be measured in image space, without considering the perspective distortion. While color scales are sufficient to enable the interpretation of color-coded information, a similar concept is not sufficient to show the meaning of glyph sizes. Only a tendency can be expressed by using such a scale.

As mentioned above, it must be also ensured that the shapes are distinguishable independent of the viewing direction. Superquadrics do not only satisfy the criterion of unambiguous perception [10], they can also be used to map a multitude of variables (see Fig. 3(a)). According to Barr's definition of superquadrics [14], they are specified besides their size based on two parameters $\alpha$ and $\beta$, which influence their roundness. In Fig. 4, an ellipsoid and a toroid superquadric shape are shown with varying $\alpha$ and $\beta$ parameters. Thus, when considering their size as another glyph property, ellipsoid superquadrics provide three degrees of freedom (DoF) for parameter mapping and toroid superquadrics provide four DoF for parameter mapping, since the radius of the tube can be additionally exploited for parameter mapping. For both cases, the glyph orientation can

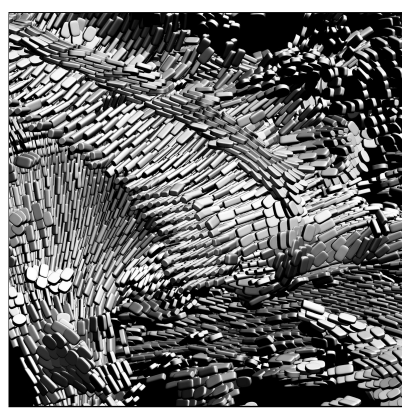

(a)

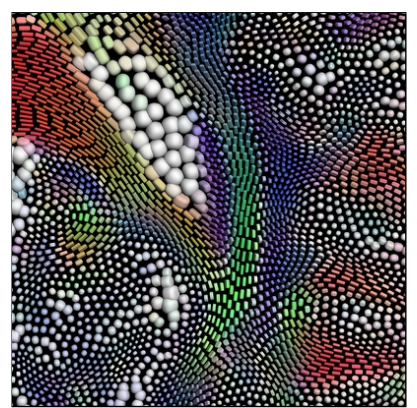

(b)

Figure 3: Superquadrics are unambiguously perceivable [10] (a). Glyphs showing diffusion directions are packed in order to remove undue visual emphasis of the regular sampling grid of the data and to illustrate larger-scale continuous features [13] (b). (Images courtesy of G. Kindlmann.)

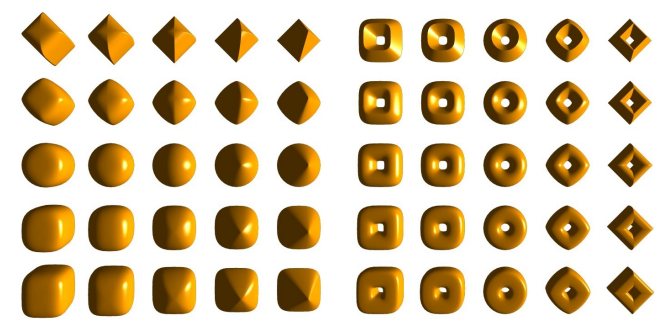

(a)

(b)
Figure 4: Superquadric glyph shapes can convey multiple variables by changing their $\alpha$ and $\beta$ parameter. This variation influences the roundness of the ellipsoid (a) and the toroid superquadric (b) along different principle directions.

be considered as additional DoF. In contrast, a spherical shape only provides a mapping with one DoF, given by the radius.

\subsubsection{Glyph Appearance}

Besides a glyph's shape, its appearance is the property most commonly used to convey information. In the context of this article, we refer to the combination of color, transparency and texture as glyph appearance. Similar to the shape-based parameter mapping, the mapping of values to the glyph appearance can also be either continuous or discrete. When using a continuous color mapping, an absolute quantification is difficult to achieve because differences in color are harder to perceive than for instance spatial distances [11]. Thus color perception can only be used to get an overview during the pre-attentive phase, i. e., local maxima and minima as well as gradients may be identified. In the following attentive phase, color scales can assist the user when interpreting the visualization. Due to the relatively high occurrence of color blindness, a color scale is preferable, which not only varies in hue, but also in luminance.

A common extension of color-coding is to employ transparency. Transparency, however, makes the perception of occlusion relations more difficult. Since occlusion is one of the strongest depth cues, and psychophysical experiments indicate that spatial perception is improved when using multiple depth cues [15], the use of transparency is expected to hamper the visual perception of objects [16]. Therefore, transparency may not be used for quantification either. In contrast it may be used 


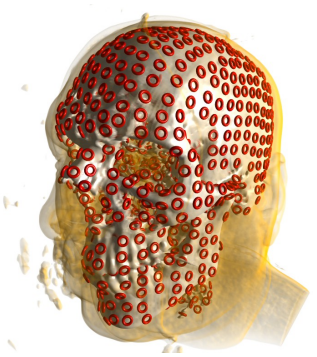

(a)

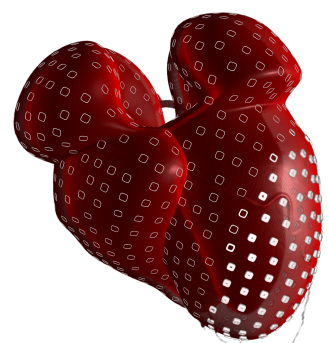

(b)
Figure 5: Feature-driven placement of glyphs within two data sets, where the glyphs are oriented according to the surface normal. Toroidal glyphs aligned to the surface of the skull (a). Glyphs depicting PET intensities as positioned on the surface of the human heart extracted from simulated CT data [12] (b). To emphasize potentially interesting regions an inverse parameter mapping is used.

to de-emphasize less important glyphs or to depict uncertainty. To communicate more complex information through the glyph appearance, as for instance directional information, also textures can be used.

\subsubsection{Glyph Placement}

To achieve a beneficial glyph visualization, also the glyph placement is crucial. Ward has already proposed a taxonomy for glyph placement strategies in the context of information visualization [1]. Some concepts of his taxonomy can be transferred to medical visualization, whereas some concepts should be omitted. In our opinion, neither a data-driven nor a structuredriven approach for glyph placement should be exploited in medical visualization (data-driven placement is based on the data dimensions and structure-driven placement on the relationship between data points), and thus both can be omitted. In contrast, in most medical applications, a reproduction strategy as described in [5] is exploited. Thus, glyphs are either placed based on the underlying regular grid, or based on the location of features present in the data set. Therefore, we distinguish between data set-driven and feature-driven placement. Placement on the regular grid is a data set-driven placement strategy, while the isosurface placement [12] is a feature-driven placement strategy (see Fig. 5). Both placement strategies are usually combined with a spatial context and may contain overlapping or non-overlapping glyphs.

Avoiding image space clustering. When choosing a data set-driven placement, the underlying structure of the regular grid usually has a major influence on the visualization. Thus it can unintentionally emphasize or even feign a non-existent glyph aggregation. However, there is a variety of techniques, which help to avoid this inadvertent effect of the underlying grid structure. Laidlaw et al. [17] proposed a jittered placement in order to reduce the aliasing introduced by the regular grid. Similarly, Meyer-Spradow et al. proposed a random distribution with relaxation, in order to get a uniform glyph distribution, when using feature-based glyph placement [4]. Bokinsky presented data-driven spots, which are used to display multiple scalar fields [18]. These spots are colored Gaussian splats, which are placed on a jittered grid. A more sophisticated ap-

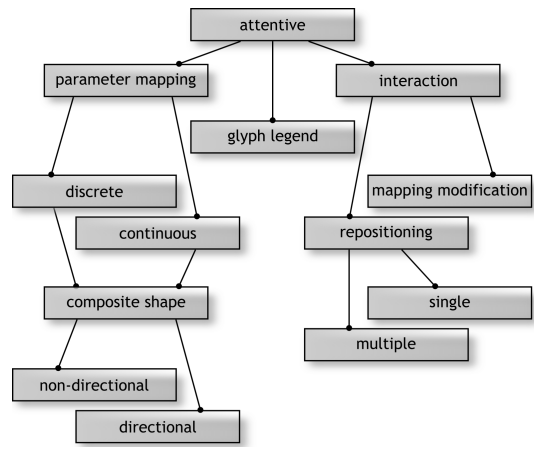

Figure 6: We consider composite glyph shapes, glyph legends and interactive techniques as the three main groups of glyph properties, which are relevant during the attentive processing.

proach has been proposed by Kindlmann and Westin [13]. They exploited a particle system in order to generate a packed glyph placement, which combines the continuous character of a texture with the used glyph technique (see Fig. 3(b)).

\subsection{Attentive Stimuli}

While the techniques described in the previous subsection primarily provide a first impression, the techniques described in this subsection allow to get into detail and possibly derive quantitative results. Thus, besides the composite glyph shapes mentioned above, we mainly focus on glyph legends and glyph interaction, which are integrated into our taxonomy as shown in Figure 6.

\subsubsection{Composite Glyph Shape}

In comparison to basic glyph shapes, composite glyph shapes (recall Section 2.1.1) may communicate more complex information in terms of dimensionality and arbitrary mappings, whereby the DoF for a parameter mapping is highly dependent on the type of glyph.

We consider directional glyphs as a subset of composite glyphs. When using directional glyphs, the semantic of the visualized data values is taken into account and is expressed by means of the glyph shape, e.g., an arrow is used to communicate a direction of movement. In medical visualization,

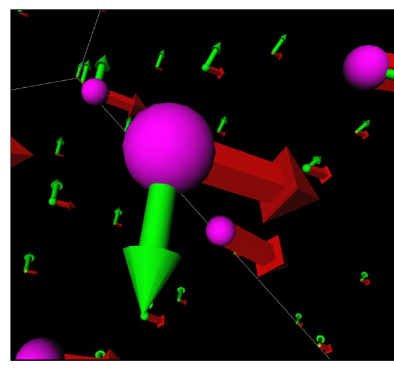

(a) Customizable glyph

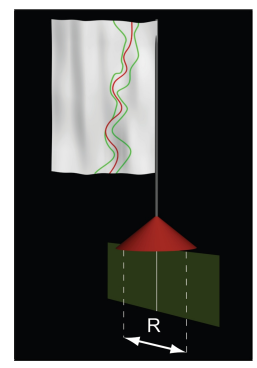

(b) Profile flag glyph
Figure 7: Composite glyphs can be customized in order to depict multiple data values [19] (a). More complex information can be visualized by integrating projection surfaces into composite glyphs [20] (b). (Images courtesy of M. Kraus and M. Mlejnek.) 
directional glyph shapes may be used to visualize blood flow or tissue movement.

Composite glyph shapes are frequently used in information visualization [21, 22]. In scientific visualization, Kraus and Ertl have proposed a system that allows non-programmers to intuitively customize composite glyphs [19]. With their system, the user can generate a composite glyph by selecting shapes from a provided set of basic shapes, and configure the orientation and scaling of each shape to be dependent on the data values. An example glyph generated using their system is shown in Fig. 7(a). In medical visualization, the profile flag glyph has been proposed as a combination of basic geometric primitives combined with a surface used to project more complex information (see Fig. 7(b)) [20]. The glyph consists of several basic primitives and allows an efficient exploration of knee MRI scans. It has a projection surface for displaying a profile through the scan, while the base cone shows which part of the scan is considered (see Fig. 7(b)). Emphasizing their attentive nature, profile flags have been proposed together with probing-like interaction metaphors: One or two glyphs are manually positioned to visualize the data value at a desired position. Further extensions of this glyph technique are described in [23].

\subsubsection{Glyph Legend}

To aid the interpretation of visually displayed information, often graphical legends are exploited. In many cases these legends are restricted to represent a color scale annotated with the according range of values. Alternatively, often symbolic maps are used as graphical legends, where a symbol is defined as a certain feature shown in the visualization. In glyph visualization, legends have to be considered as a hybrid between graphical and symbolic legends. On the one hand, the legend should allow the user to mentally reconstruct the parameter mapping, on the other hand, it might be also helpful to display glyphs for certain values which define an important threshold. Thus, with such a legend, the user is able to visually compare the glyphs shown in the current visualization with characteristic glyphs included in the legend [12] (see Fig. 8). However, since glyphs need to be visually matched with the glyph legend, absolute quantification is still difficult. Additionally, when certain parameters are mapped to a glyph's size, this might be influenced by the perspective distortion, making the visual matching of equally sized glyphs contained in the legend even more difficult. A typical glyph legend is given by multiple rows, each depicting the range of values for one glyph property. Thus, a glyph legend implicitly depicts the DoF of the used PMF.

\subsubsection{Glyph Interaction}

We consider the probing-like repositioning of glyphs as well as the interactive modification of the parameter mapping as the most important interactions influencing the attentive processing of glyph visualizations.

Repositioning glyphs. Many glyph visualization techniques exist, where the initial glyph placement can be interactively modified by moving one or two proxy glyphs through the data set $[20,24]$. These glyphs, which are also referred to as probing tools [25], adapt their visual appearance based on the PMF to the new location they are moved to. Thus, repositioning glyphs can be compared to using a color picker tool.

Sigfridsson et al. have proposed such glyphs for tensor field visualization, where the glyphs can be positioned within a continuous field representation, in order to get quantitative values at the desired position [26]. A more complex probing glyph, is the previously mentioned profile flag glyph described in [20] (recall Fig. 7(b)). To allow a semi-quantitative analysis, two profile flags can be visualized and repositioned simultaneously such that their visualization is comparable. Thus, it has to be distinguished between single and multiple probing glyphs, which can be repositioned.

Parameter mapping modification. In comparison to reposition individual glyphs, interactively modifying the PMF is much less frequently considered in the literature. Only a few efforts have been undertaken in order to support interactive parameter mapping [27]. Instead, most researchers have focussed on how glyphs can be visualized when a certain parameter mapping is present $[19,10,28,12]$. However, generating such a parameter mapping is crucial and should also be interactive. Especially because different glyph representations may be developed with the goal to emphasize certain features. With glyph filtering, it is possible to display only glyphs, which satisfy a certain selection criterion. For instance, in the context of diffusion tensor imaging (DTI) visualization, it would be possible to visualize only glyphs exceeding a certain level of fractional anisotropy. Another example would be blood flow visualization, where it might be desirable to exclude glyphs representing values above a certain speed level. In all cases, filtering has a major influence on the overall glyph distribution. Modifying this glyph distribution can be used to direct the user's attention, or to emphasize critical data values. Thus, glyph filtering could alternatively be also considered as a glyph placement strategy, since less meaningful glyphs are omitted from display. While most filtering techniques are performed automatically by the system, the user should also have the possibility to filter glyphs by changing the PMF.

\section{Applications}

In this section, we describe medical application areas, in which glyph-based visualization techniques are exploited. The goal is not to give a comprehensive overview of the described glyph techniques. Instead, we pick out DW-MRI and cardiac visualization as prominent examples. An overview on stress and strain tensor glyphs in non-medical domains can be found in [29].

Table 1 gives an overview of the applications described in this section. It relates the techniques to our taxonomy, by listing their most important properties, as the modality of the visualized data, the used glyph shapes, the glyph placement and the used parameter mapping.

\subsection{Diffusion Weighted MRI Visualization}

In medical visualization, DW-MRI is probably the domain where the usage of glyphs has been most intensively investigated. DW-MRI exploits that the diffusion rate of water 


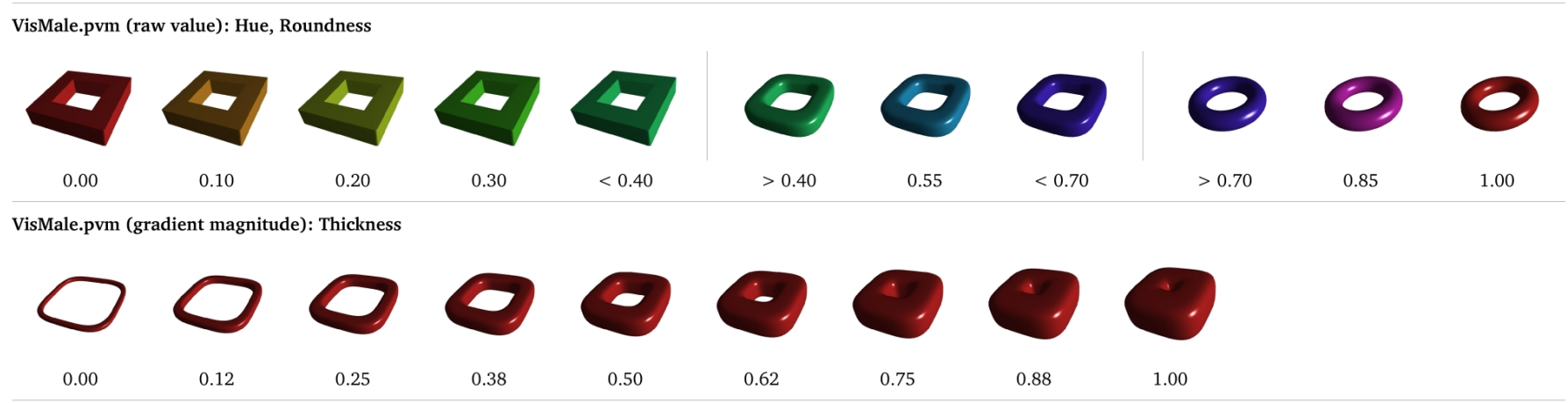

Figure 8: Glyph legends help with the interpretation of a glyph visualization [12]. Legends can be especially helpful when dealing with discontinuous mapping functions as shown in the two bottom rows of Figure 2.

Table 1: A listing of the most important glyph properties used in the applications described in Section 3: the modality of the visualized data, the used glyph shapes, their placement and the used parameter mapping.

\begin{tabular}{c|l|l|l|c} 
Modality & Glyph Shape & Glyph Placement & Parameter Mapping & Reference \\
\hline DTI & spherical harmonics & grid & higher order tensor to shape & {$[30]$} \\
DTI & HOME & - & higher order tensor to shape & {$[31]$} \\
DTI & ellipsoids & grid & one tensor value to glyph size & {$[17]$} \\
DTI & stroke layers & random & all tensor value to shape and color & {$[17]$} \\
DTI & superquadrics & grid & eigenvector and eigenvalue to shape and orientation & {$[10]$} \\
DW-MRI & deformed spheres & grid & deformation depicts diffusion direction & {$[28]$} \\
DTI & ellipsoids & interactive & eigenvector and eigenvalue to shape & {$[26]$} \\
DTI & lines & interactive & eigenvector and eigenvalue to length and direction & {$[26]$} \\
DTI & superquadrics & packing & eigenvector to color & {$[13]$} \\
DTI & Gabor patches & grid & eigenvector to color, anisotropy to transparency & {$[32]$} \\
DW-MRI & ellipsoids & grid & deformation to color & {$[33]$} \\
PET/CT & superquadrics & feature-driven & interactive & {$[12]$} \\
MRI & cuboids \& custom & data set-driven & peak enhancement to color, Up Slope to size & {$[34]$}
\end{tabular}

molecules allows to derive information about the structures of the underlying tissue. However, glyphs are only suitable for getting a first impression immediately after the acquisition. Glyph visualizations cannot be exploited during a neurosurgical intervention since glyphs do not explicitly show the underlying fibers which must not be affected during an intervention.

Laidlaw et al. have proposed glyph techniques for the representation of DTI data derived from the mouse spinal cord [17]. They exploited an array of ellipsoids, where the shape of the ellipsoids present one tensor value, whereas their size is equal due to an introduced normalization. Their second technique used multiple layers of varying brush strokes, to represent all tensor values. The authors state that the ellipsoids are easier to interpret, while the brush stroke visualization is more quantitative. Integrated approaches of stream- and glyph-based techniques have been proposed by Hlawitschka and Scheuermann [30] as well as Chen et al. [35].

Kindlmann proposed superquadric glyph shapes to convey the principal eigenvectors of a diffusion tensor in order to depict the microstructure of white-matter tissue of the human brain [10]. The distinct glyphs are placed at a regular grid and controlled by a fractional anisotropy threshold in order to min- imize visual clutter. Jankun-Kelly et al. [36] have evaluated the use of four different glyph visualizations for depicting traceless tensor data. They could show, that among the tested techniques, superquadric glyphs led to lower total error and lower response times. Their approach, that has been originally developed for nematic liquid crystal alignment tensors, is not based on the offsets of the eigenvalues, but on physically-linked metrics.

Domin et al. criticize that most glyphs proposed for DTI visualization are not sufficient [28]. The major drawback is that the used glyphs cannot convey the possibly arbitrary diffusion directions, and the data is usually reduced to $6 \mathrm{DoF}$ in the modeling stage. Several approaches avoid this limitation by scaling vectors on the sphere based on the diffusion coefficients [37, 38, 28]. Fig. 9(a) shows the approach by Domin et al. [28]. However, it should be investigated how far the deformed sphere geometry can be perceived without introducing a cognitive overload.

Sigfridsson et al. have presented a hybrid approach for visualizing tensor fields [26]. Their approach integrated an overview of the field, which is generated through adaptive filtering (see Fig. 9(b)). While this provides the context, glyphs can be used in the attentive phase in order to get more detailed 
information. Therefore, the glyphs can be positioned freely.

To avoid the perception of false glyph aggregation in DTI data, Kindlmann and Westin have proposed a glyph packing algorithm [13] that exploits a modified particle-system (recall Fig. 3(b)). Hlawitschka et al. have also proposed a packing algorithm for the same application case [24] that achieves fast clustering. However, they describe the packing on a single slice only, which can be moved through the volume.

Since DTI data are primarily analyzed with respect to the direction of principal diffusion, it is reasonable to use transparency to convey the amount of anisotropy [32]. With this strategy, directional information is only pronounced if it is reliable. The use of transparency requires some background information, either a constant background color or anatomic information, such as a T2-weighted image (see Fig. 10(a)). Benger et al. also employ the human shape perception capabilities in particular with respect to pattern discrimination [32]. As a general strategy, they suggest to map tensorial quantities to texture patterns. More specifically, they employ the Gabor filter (see Fig. 10(b) and [39]), which conveys directional information well. A Gabor patch is mapped to the plane formed by the principal and median eigenvector (the $y$-direction of the Gabor patch is aligned with the principal eigenvector). Mapping tensor information to Gabor textures is optionally combined with the previously defined mapping to color and transparency. Thus, anisotropy characteristics are mapped to color, transparency, and texture (see Fig. 10(b)), while directional information is only visualized if it is assessed as reliable based on the relation between the eigenvalues.

\subsection{Higher-Order Tensor Glyphs}

While most DTI visualization approaches focus on secondorder tensor fields derived from MRI data, some techniques have been also developed for higher-order tensor data [40, 41, 42, 43]. Hlawitschka and Scheuermann presented a technique for higher order tensor fields [30]. They exploit the analogy of higher order tensors and spherical harmonics, and propose a glyph visualization inspired by this analogy. Thus, the authors generate glyphs of symmetric fourth order tensors by deforming a subdivided icosahedron based on the pre-computed spherical harmonic representation. Schultz and Kindlmann have presented another glyph approach for higher-order tensors [31].

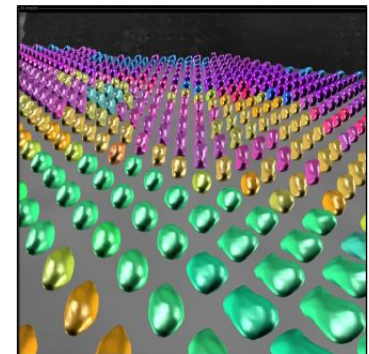

(a) Deformed spheres

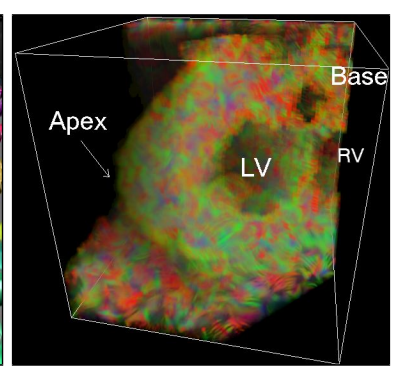

(b) Probing glyphs
Figure 9: Deformed spheres are used to visualize diffusion directions [28] (a). Glyphs can be positioned within a continuous tensor field representation [26] (b). (Images courtesy of L. Linsen and A. Sigfridsson.)

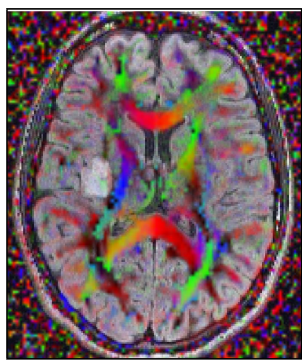

(a) The components of the principal eigenvector are mapped to the red-, greenand blue-component of color. Transparency indicates linear anisotropy.

Figure 10: Usage of color and transparency for DTI visualization. (Images courtesy of W. Benger.)

The proposed glyphs are colored based on the parameters to be visualized and allow to emphasize the depicted maxima values by introducing sharp edges, at the cost of smoother shapes around the minima. Therefore, the proposed glyphs are referred to as higher-order maximum enhanced (HOME) glyphs. A composite DTI glyph has been also proposed by Westin et al. [44]. For each glyph, they combine a sphere, a disc and a rod. The radius of the disc is determined based on the largest eigenvalue, while the radius of the disk is determined by the second largest eigenvalue. Finally, the length of the rod is set to twice the largest eigenvalue.

\subsection{Cardiac Visualization}

In this section, we address glyph-based visualizations for assessing functional and structural parameters of the heart. Choi et al. present such glyphs specifically designed for cardiac visualization [45]. They propose a technique for accurately measuring ventricular volume, mass, wall thickness, and wall motion, based on a $3 \mathrm{D}$ shape reconstruction through fitting a deformable model. The data extractable from the model can be visualized by using glyph techniques also in a quantitative manner. With their technique, different variables can be mapped to properties of the glyph allowing a comprehensible visualization of these multivariate data sets.

Wünsche and Lobb introduced a glyph-based technique to visualize wall motion of the heart [33]. Based on a generated finite element model, they position ellipsoid glyphs to show the movement of the myocardium. Each glyph is divided into six regions, whereas the region color encodes whether a dilation or a contraction is present.

Ropinski et al. have proposed easily modifiable superquadric glyphs which they also apply to cardiac visualization [12]. With their surface-based placement strategy, they are able to position the glyphs directly on the myocardium. Thus, the high resolution CT data provides the morphological context, while the low resolution PET data is depicted by using the glyphs (see Fig. 5). By choosing appropriate PMFs, it is possible to guide the user's 


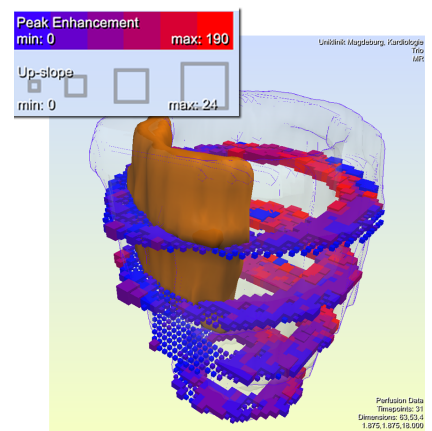

(a) Grid placement

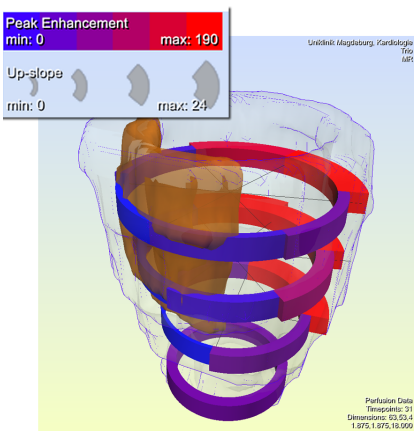

(b) 17-segment model placement
Figure 11: Glyph visualizations developed to support the analysis of cardiac MR data. Perfusion parameters are encoded voxel-wise by cuboid glyphs (a) or segment-wise by a more sophisticated glyph shape (b) [34].

attention to regions of interest, e.g., areas of the myocardium with low PET activity.

Oeltze et al. exploited glyphs to visualize cardiac MRI data in order to also allow an exploration of the structure and the function of the myocardium [34]. Cuboid-shaped glyphs map two parameters describing the perfusion of the myocardium [46]: peak enhancement to color and Up-Slope to size. The glyphs are placed voxel-wise for each slice of the perfusion scan and are integrated in a visualization of the left ventricle and scar tissue derived from MRI late enhancement data (see Fig. 11(a)). Glyphs may also be aggregated according to the 17-segment model of the American Heart Association (AHA). A more sophisticated glyph shape represents a segment of the myocardium (see Fig. 11(b)). Another approach has been presented by Ennis et al. [47]. They have described the use of superquadric glyphs, which allow a better differentiation of the fiber direction as compared to ellipsoidal glyphs.

\section{Usage Guidelines}

In this section we propose guidelines for the usage of glyph techniques. It should be mentioned that apart from the work by Spradow et al. [4] and Jankun-Kelly et al. [36] to our knowledge no other formal evaluation of glyph techniques has been conducted so far. Therefore, these guidelines should rather be considered as conjectures derived from the best practice described in the literature. We do not state that these guidelines are complete or describe a theory, but they distill the observations, we have made during our literature review. We believe they can be a helpful condensation for the interested reader. Therefore, we have derived the following six usage guidelines for the integration of glyph-based techniques in medical visualization, which in our opinion reflect the current state of the art:

\section{Parameter mapping functions should}

- visually emphasize important variables.

- incorporate the range of values.

- guide the user's focus of attention to encode relevance.
- incorporate semantics of the data.

- be mentally reconstructable based on the visualization.

2. Glyph placement should be well-balanced and avoid unwanted glyph aggregations in image space, e. g., by applying jittering or relaxation procedures.

3. Glyph shapes should be unambiguously perceivable independent of the viewing direction.

4. Glyph visualizations should support quantitative analysis in the attentive phase.

5. Hybrid visualization should be exploited to provide the anatomical context.

6. When using the glyph size to convey information, perspective projections should be avoided.

Most of these guidelines are focused on choosing an appropriate PMF, which is crucial to allow comprehensive glyph visualizations. According to the first guideline, the most important variables should be more prominent in the final visualization. When, for instance, using a torus-like glyph, the color is better perceivable than the roundness of the glyph. Since the range of values present in a data set has a major impact on the right choice of a mapping function, this range should be considered carefully when specifying the function. Furthermore, an appropriate parameter mapping can also be used to guide the user's focus of attention. In Fig. 5, an inverse parameter mapping is used, i. e., low PET activity is mapped to thick glyphs, while high PET activity is mapped to thin glyphs. By using this inverse mapping, the region of interest, namely the region with reduced PET activity, is visually emphasized. Additionally, the parameter mapping should be intuitive, i. e., in cases where a glyph property fits semantically to a parameter to be mapped, it should be assigned to this parameter. For instance, in cases where a parameter represents the dimensions of a feature, it should be mapped to the glyph's size. Intuitive mappings are presented in [48].

Also colors should be chosen wisely when specifying a color PMF. In some applications, widely accepted color mappings are present. These should be considered when exploiting glyph techniques. For instance, PET data sets are often visualized exploiting a heat map, i. e., a yellow-to-red gradient. In cases, where no widely accepted color mapping is available, the mapping should be chosen by considering color perception, and eventually the semantics of the variables to be visualized. When taking into account the opponent color model [49], two-colored gradients can be generated, whereas the two colors are perceived as lying along the opposite directions of a coordinate axis. According to the opponent color theory, the three color axis are specified by red and green, blue, and yellow as well as black and white. However, it should also be considered that the displayed colors influence spatial comprehension. The chromadepth technique [50] supports depth perception based on the fact that the lens of the eye refracts colored light with different wavelengths at different angles. Although this effect can be supported by diffraction grating glasses, watching images without instrumentation can also result in a depth effect. 
Furthermore, for all parameter mappings it is important that the mapping supports a mental interpolation, i. e., the user is able to mentally reconstruct the PMF when viewing distinct glyphs. This can be supported when equal perceptual distances match distances in the range of values which is true when specifying colors in the CIE color model.

As expressed by the second guideline, glyph placement is also important for the comprehension of glyph-based visualizations. Since a regular placement on the grid may convey non-existent aggregations unintendedly $[12,13]$, this has to be avoided. Therefore, when using a data set-driven placement in combination with regular grids often occurring in medical imaging data, at least a jittering has to be applied [17]. Moreover, when a texture-like appearance is desired, glyph packing strategies are sufficient in order to avoid misleading aggregations [13]. In general, the placement should be chosen in a way that the observer can perceive existent aggregations easily. Thus, also the glyph size and the question whether glyphs are overlapping or non-overlapping have to be taken into account. Since the glyph size and thus the spacing between adjacent glyphs is dependent on the resolution of the data set, general guidelines cannot be proposed.

Independently of parameter mapping and placement, the used glyph shapes should also satisfy certain criteria to allow a comprehensible visualization. First of all, glyph shapes should be unambiguously perceivable independent of the viewing direction as stated by the third guideline. Another important criterion possibly resulting in improved perception is the usage of intuitive glyph shapes. Similar to choosing an intuitive parameter mapping, a glyph shape can be chosen which represents the semantics of the variables to be shown. For instance, when considering tissue motion or blood flow direction, directional glyphs such as arrows are sufficient.

While the previous guidelines are focussed on the preattentive phase, in some cases a quantitative analysis in the attentive phase is desired. For these application cases, interaction metaphors, e. g., probing tools [26], and glyph legends [12] should be integrated, as expressed by the fourth guideline.

Furthermore, especially when choosing a rather large glyph spacing and thus a lot of context would become visible, the fifth guideline should be considered, i.e., the visualization should be enhanced by integrating a spatial context, for instance, by visualizing morphological structures through rendering selected structures from a CT data set. Since such a hybrid visualization is often described in literature, dealing with glyph-based medical visualization $[12,26]$, it can be assumed as helpful in many cases.

Finally, in order to support the quantification in the attentive phase, according to the sixth guideline, perspective projections should be avoided, when mapping parameters to glyph size. Instead an orthographic projection ensures that glyphs are quantifiable and the user can visually compare glyphs to glyphs at different locations or in the legend.

As mentioned above, all these guidelines have been derived from the best practice. Thus, they just reflect the current state of the art, and have to be subject to a systematic evaluation in the near future. However, although these guidelines have been derived from applications within the area of medical visualization, they may also be transferable to a certain extent to other domains.

\section{Conclusions and Future Work}

In this article we have described a taxonomy for glyph properties to be used in medical visualizations. In our taxonomy we classify these properties by considering aspects from the area of perception. Thus, we were able to identify glyph properties supporting pre-attentive and attentive visual processing. This distinction is important, since medical diagnoses are often performed under time pressure, and thus it is essential that the most relevant information is conveyed in a very direct way. Based on our literature review, we have proposed six guidelines with the goal to support improved glyph-based visualizations in the future. Since these guidelines are only derived from observations, a systematic evaluation is necessary in order to prove their usefulness. However, our taxonomy and to some extent also the guidelines have been motivated by the feature integration theory of attention. The pre-attentive processing is known to be partially dependent on the experiment setup that would be in our case equivalent to the glyph design. For instance, it is known that the expectation of the viewer as well as the similarity of the distracters have an influence on the pre-attentive phase [51, 52]. Furthermore, in some cases the existence of a certain feature is better perceivable within a group of objects not having this feature, than its absence within a group having this feature [53]. The latter is not an issue when only two variables are depicted by the shown glyphs. Nevertheless, when multiple variables are depicted, the distracters might not look similar enough to fully support pre-attentive processing. To be able to conduct the previously mentioned evaluation, all these aspects need to be taken into account.

\section{Acknowledgments}

We thank Christian Döring, Arvid Malyczyk, Jennis MeyerSpradow, Lydia Paasche and Michael Specht for contributing some of the techniques described in this paper. Furthermore, we thank the reviewers for the very fruitful comments, which added great value to this paper. Finally, we thank all authors for permitting to use their images. This work was supported by the DFG Priority Program 1335: Scalable Visual Analytics.

\section{References}

[1] M. O. Ward, A taxonomy of glyph placement strategies for multidimensional data visualization, Information Visualization 1 (3) (2002) 194-210.

[2] R. Bürger, H. Hauser, Visualization of multi-variate scientific data, in: EuroGraphics State of the Art Reports (STARs), 2007, pp. 117-134.

[3] T. Ropinski, B. Preim, Taxonomy and usage guidelines for glyph-based medical visualization, in: Proc of Simulation and Visualization, 2008, pp. 121-138.

[4] J. Meyer-Spradow, L. Stegger, C. Döring, T. Ropinski, K. H. Hinrichs, Glyph based spect visualization for the diagnosis of coronary artery disease, IEEE Transactions on Visualization and Computer Graphics (TVCG) (Vis Conference Issue) (2008) 1499-1506. 
[5] J. F. J. Rodrigues, A. J. M. Traina, M. C. F. de Oliveira, C. J. Traina, Reviewing data visualization: an analytical taxonomical study, in: Information Visualization, 2006, pp. 713-720.

[6] C. Ware, Information visualization: perception for design, Morgan Kaufmann Publishers Inc., 2000.

[7] A. Treisman, Preattentive processing in vision, Comput. Vision Graph. Image Process. 31 (2) (1985) 156-177.

[8] J. R. Anderson, Cognitive Psychology and its Implications, 6th Edition, Worth Publishers, 2004.

[9] B. Shneiderman, The eyes have it: A task by data type taxonomy for information visualizations, in: IEEE Symposium on Visual Languages, 1996, p. 336.

[10] G. L. Kindlmann, Superquadric tensor glyphs., in: Joint Eurographics IEEE TCVG Symposium on Visualization, 2004, pp. 147-154.

[11] W. S. Cleveland, R. McGill, Graphical Perception: Theory, Experimentation, and Application to the Development of Graphical Methods, Journal of the American Statistical Association 79 (387) (1984) 531-554.

[12] T. Ropinski, M. Specht, J. Meyer-Spradow, K. H. Hinrichs, B. Preim, Surface glyphs for visualizing multimodal volume data, in: International Fall Workshop on Vision, Modeling, and Visualization (VMV07), 2007, pp. 3-12.

[13] G. Kindlmann, C.-F. Westin, Diffusion tensor visualization with glyph packing, IEEE Transactions on Visualization and Computer Graphics 12 (5) (2006) 1129-1135.

[14] A. H. Barr, Superquadrics and angle-preserving transformations, IEEE Computer Graphics and Applications 1 (1) (1981) 11-23.

[15] N. Bruno, J. Cutting, Minimodularity and the perception of layout, Journal of Experimental Psychology 117 (2) (1988) 161-170.

[16] V. Interrante, H. Fuchs, S. M. Pizer, Conveying the $3 \mathrm{~d}$ shape of smoothly curving transparent surfaces via texture, IEEE Transactions on Visualization and Computer Graphics 3 (2) (1997) 98-117.

[17] D. H. Laidlaw, E. T. Ahrens, D. Kremers, M. J. Avalos, R. E. Jacobs, C. Readhead, Visualizing diffusion tensor images of the mouse spinal cord, in: IEEE Visualization, 1998, pp. 127-134.

[18] A. Bokinsky, Multivariate data visualization with data-driven spots, $\mathrm{Ph} . \mathrm{D}$. thesis, University of North Carolina - Chapel Hill (2003).

[19] M. Kraus, T. Ertl, Interactive data exploration with customized glyphs, in: WSCG, 2001, pp. 20-23.

[20] M. Mlejnek, P. Ermes, A. Vilanova, R. van der Rijt, H. van den Bosch, F. Gerritsen, E. Gröller, Profile flags: a novel metaphor for probing of T2 maps, in: IEEE Visualization, 2005, pp. 599-606.

[21] D. S. Ebert, R. M. Rohrer, C. D. Shaw, P. Panda, J. M. Kukla, D. A Roberts, Procedural shape generation for multi-dimensional data visualization, in: Data Visualization, 1999, pp. 3-12.

[22] M. D. Lee, R. E. Reilly, M. E. Butavicius, An empirical evaluation of chernoff faces, star glyphs, and spatial visualizations for binary data, in: Asia-Pacific Symposium on Information visualisation, 2003, pp. 1-10.

[23] M. Mlejnek, P. Ermes, A. Vilanova, R. van der Rijt, H. van den Bosch, E. Gröller, F. Gerritsen, Application-oriented extensions of profile flags, in: EG/IEEE VGTC Symposium on Visualization, 2006, pp. 339-346.

[24] M. Hlawitschka, G. Scheuermann, B. Hamann, Interactive glyph placement for tensor fields, in: Advances in Visual Computing, Third International Symposium, ISVC, 2007, pp. 331-340.

[25] L. Hong, X. Mao, A. Kaufman, Interactive visualization of mixed scalar and vector fields, in: IEEE Visualization, 1995, pp. 240-247.

[26] A. Sigfridsson, T. Ebbers, E. Heiberg, L. Wigström, Tensor field visualization using adaptive filtering of noise fields combined with glyph rendering, in: IEEE Visualization, 2002, pp. 371-378.

[27] M. van Almsick, T. H. Peeters, V. Prckovska, A. Villanova, B. ter Haar Romeny, Gpu-based ray-casting of spherical functions applied to high angular resolution diffusion imaging, IEEE Transactions on Visualization and Computer Graphics 15 (1).

[28] M. Domin, S. Langner, N. Hosten, L. Linsen, Direct glyph-based visualization of diffusion MR data using deformed spheres, in: Visualization in Medicine and Life Sciences, 2007.

[29] Y. Hashash, J. Yao, D. Wotring, Glyph and hyperstreamline representation of stress and strain tensors and material constitutive response, International Journal of Numerical and Analytical Methods in Geomechanics 27 (2003) 603-626.
[30] M. Hlawitschka, G. Scheuermann, Hot- lines: Tracking lines in higher order tensor fields, in: IEEE Visualization, 2005, pp. 27-34.

[31] T. Schultz, G. Kindlmann, A maximum enhancing higher-order tensor glyph, Computer Graphics Forum 29 (3) (2010) 1143-1152.

[32] W. Benger, H. Bartsch, H.-C. Hege, H. Kitzler, A. Shumilina, A. Werner, Visualizing Neuronal Structures in the Human Brain via Diffusion Tensor MRI, International Journal of Neuroscience (2006) 461-514.

[33] B. Wünsche, R. Lobb, A. Young, The visualization of myocardial strain for the improved analysis of cardiac mechanics, in: GRAPHITE, 2004, pp. $90-99$.

[34] S. Oeltze, A. Hennemuth, S. Glaer, C. Khnel, B. Preim, Glyph-based visualization of myocardial perfusion data and enhancement with contractility and viability information, in: EG Workshop on Visual Computing for Biology and Medicine, 2008, pp. 11-20.

[35] W. Chen, S. Zhang, S. Correia, D. F. Tate, Visualizing diffusion tensor imaging data with merging ellipsoids, in: IEEE Pacific Visualization Symposium, 2009, pp. 145-151.

[36] T. J. Jankun-Kelly, Y. S. Lanka, J. E. Swan II, An evaluation of glyph perception for real symmetric traceless tensor properties, Computer Graphics Forum 29 (3) (2010) 1133-1142.

[37] A. L. Alexander, K. M. Hasan, M. Lazar, J. S. Tsuruda, D. L. Parker, Analysis of partial volume effects in diffusion-tensor MRI, Magnetic Resonance in Medicine 45 (2001) 770-780.

[38] L. R. Frank, Anisotropy in high angular resolution diffusion-weighted MRI, Magnetic Resonance in Medicine 45 (2001) 935-939.

[39] M. Porat, Y. Y. Zeevi, The generalized gabor scheme of image representation in biological and machine vision, IEEE Trans. Pattern Anal. Mach. Intell. 10 (4) (1988) 452-468.

[40] L. R. Frank, Characterization of anisotropy in high angular resolution diffusion-weighted MRI, Magnetic Resonance in Medicine 47 (2002) 1083-1099.

[41] D. C. Alexander, G. J. Barker, S. R. Arridge, Detection and modeling of non-gaussian apparent diffusion coefficient profiles in human brain data, Magnetic Resonance in Medicine 48 (2002) 331-340.

[42] E. Özarslan, T. Mareci, Generalized diffusion tensor imaging and analytical relationships between diffusion tensor imaging and high angular resolution diffusion imaging, Magnetic Resonance in Medicine 50 (2003) 955-965.

[43] J.-D. Tournier, F. Calamante, D. G. Gadian, A. Connelly, Direct estimation of the fiber orientation density function from diffusion-weighted MRI data using spherical deconvolution, NeuroImage 23 (2004) 1176-1185.

[44] C.-F. Westin, S. Maier, H. Mamata, A. Nabavi, F. Jolesz, R. Kikinis, Processing and visualization for diffusion tensor MRI, Medical Image Analysis 6 (2002) 93-108.

[45] S. Choi, D. Lee, S.-J. Yoo, M.-H. Kim, Interactive visualization of diagnostic data from cardiac images using 3D glyphs., in: ISMDA, 2003, pp. 83-90.

[46] B. Preim, S. Oeltze, M. Mlejnek, E. Gröller, A. Hennemuth, S. Behrens, Survey of the visual exploration and analysis of perfusion data, IEEE Transactions on Visualization and Computer Graphics 15 (2) (2009) 205220.

[47] D. B. Ennis, G. Kindlmann, I. Rodriguez, P. A. Helm, E. R. McVeigh, Visualization of tensor fields using superquadric glyphs, Magnetic Resonance in Medicine 53 (1) (2005) 169-176.

[48] S. Oeltze, A. Malyszczyk, B. Preim, Intuitive mapping of perfusion parameters to glyph shape, in: Bildverarbeitung für die Medizin (BVM 2008), 2008, pp. 262-266.

[49] M. Bollmann, B. Mertsching, Opponent color processing based on neural models, in: International Workshop on Advances in Structural and Syntactical Pattern Recognition, 1996, pp. 198-207.

[50] R. Steenblik, The chromostereoscopic process: A novel single image stereoscopic process, in: Proc of SPIE - True 3D Imaging Techniques and Display Technologies, 1987.

[51] J. Duncan, Boundary conditions on parallel search in human vision, Perception 18 (1989) 457-469.

[52] J. Duncan, G. Humphreys, Visual search and stimulus similarity, Psychological Review 96 (3) (1989) 433-458.

[53] A. Treisman, Properties, parts and objects, Wiley, 1986 